Draft VERsion April 8, 2019

Preprint typeset using $\mathrm{LAT}_{\mathrm{E}} \mathrm{X}$ style AASTeX6 v. 1.0

\title{
DISCOVERY OF A COMPACT COMPANION TO A NEARBY STAR
}

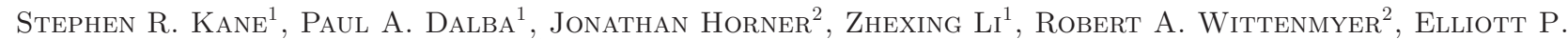 \\ $\mathrm{Horch}^{4}$, Steve B. Howell ${ }^{3}$, Mark E. EveretT ${ }^{5}$
}

\footnotetext{
${ }^{1}$ Department of Earth and Planetary Sciences, University of California, Riverside, CA 92521, USA

${ }^{2}$ Centre for Astrophysics, University of Southern Queensland, Toowoomba, QLD 4350, Australia

${ }^{3}$ NASA Ames Research Center, Moffett Field, CA 94035, USA

${ }^{4}$ Department of Physics, Southern Connecticut State University, New Haven, CT 06515, USA

${ }^{5}$ National Optical Astronomy Observatory, 950 N. Cherry Ave, Tucson, AZ 85719, USA
}

\begin{abstract}
Radial velocity (RV) searches for exoplanets have surveyed many of the nearest and brightest stars for long-term velocity variations indicative of a companion body. Such surveys often detect highamplitude velocity signatures of objects that lie outside the planetary mass regime, most commonly those of a low-mass star. Such stellar companions are frequently discarded as false-alarms to the main science goals of the survey, but high-resolution imaging techniques can be employed to either directly detect or place significant constraints on the nature of the companion object. Here, we present the discovery of a compact companion to the nearby star HD 118475. Our Anglo-Australian Telescope (AAT) RV data allow the extraction of the full Keplerian orbit of the companion, found to have a minimum mass of $0.445 M_{\odot}$. Follow-up speckle imaging observations at the predicted time of maximum angular separation rule out a main-sequence star as the source of the RV signature at the $3.3 \sigma$ significance level, implying that the companion must be a low-luminosity compact object, most likely a white dwarf. We provide an isochrone analysis combined with our data that constrain the possible inclinations of the binary orbit. We discuss the eccentric orbit of the companion in the context of tidal circularization timescales and show that non-circular orbit was likely inherited from the progenitor. Finally, we emphasize the need for utilizing such an observation method to further understand the demographics of white dwarf companions around nearby stars.

Keywords: white dwarfs - techniques: radial velocities - techniques: high angular resolution - stars: individual (HD 118475)
\end{abstract}

\section{INTRODUCTION}

Over the last decade, large-scale exoplanet surveys have become increasingly common. Whilst transit surveys, such as Kepler (Borucki et al. 2010) and TESS (Ricker et al. 2015) are focused on the search for small planets orbiting close to their host stars, a number of long-term radial velocity (RV) surveys continue to scour the sky, achieving particular sensitivity to long-period giant planets (Bonfils et al. 2013; Butler et al. 2017), analogous to the solar system's Jupiter and Saturn (Wittenmyer et al. 2016). Since RV semi-amplitudes decrease toward longer periods, the sensitivity of RV surveys likewise shifts towards high masses with increasing orbital period (Kane et al. 2007). Even though this limits the use of such surveys in exploring the low-mass, distant planet regime, they remain ideally suited as probes

skane@ucr.edu of the occurrence of objects that bridge the gap between the planetary and stellar mass regimes. Such surveys are therefore ideal for the study of the demographics in that region (Duquennoy \& Mayor 1991; Raghavan et al. 2010). Combining RVs with high angular resolution observations provides an additional avenue through which we can test models of the frequency of companion mass for a variety of stars (Kane et al. 2014; Crepp et al. 2016; Wittrock et al. 2017), including stars known to host exoplanets (Kane et al. 2015; Roberts et al. 2015; Wittrock et al. 2016).

The combination of imaging and RV techniques can further be used to detect more exotic companions, such as compact objects. An example of this is the detection of a white dwarf that was first identified through the observation of a linear trend in the RVs measured for HD 169889, before being directly observed using high-contrast imaging (Crepp et al. 2018). Numerous RV observations of white dwarfs have been carried 
out (Barnbaum \& Zuckerman 1992; Maxted et al. 2000; Rebassa-Mansergas et al. 2017), but it is relatively rare for white dwarfs to be identified using the RV method due to the ambiguity of the orbital inclination.

The Anglo-Australian Planet Search (AAPS) is one of the longest running RV searches for exoplanets, with a temporal baseline of 18 years (Wittenmyer et al. 2014). The results from this survey have revealed numerous high-amplitude RV signatures that fall outside of the planetary mass regime. Here, we present RV data for the star HD 118475 that reveal a companion moving on a well constrained 2070 day period orbit. The minimum mass of the companion, $0.445 M_{\odot}$, would place it firmly in the stellar mass regime - comparable to the mass of an early $\mathrm{M}$ dwarf. As the primary star is relatively nearby $(\sim 33 \mathrm{pcs})$, the orbital separation of the secondary ( $\sim 3.69 \mathrm{AU})$ corresponds to an angular separation of $0.11^{\prime \prime}$ at quadrature. Despite the fact that such a companion should be readily detected, follow-up observations carried out close to the predicted maximum angular separation using the Differential Speckle Survey Instrument (DSSI) on Gemini-South rule out such a main-sequence star as the companion. As a result, we conclude that the companion must instead be a compact object, such as a white dwarf.

The structure of this paper is laid out as follows. In Section 2 we provide the RV data along with the best-fit Keplerian orbital solution. Section 3 includes the details of the DSSI observations and the reduced data confirming the exclusion of a bright companion. In Section 4, the significance of a null detection is quantified in the context of stellar isochrones, and we use the compact nature of the companion to place additional constraints on the orbital inclination of the system. We provide concluding remarks in Section 5 along with suggestions for further observations.

\section{COMPANION MASS AND ORBIT}

The RV observations of HD 118475 were acquired using the UCLES high-resolution spectrograph (Diego et al. 1990) on the $3.9 \mathrm{~m}$ Anglo-Australian Telescope (AAT). The observations and data reduction are able to routinely achieve a velocity precision of 2-3 $\mathrm{m} \mathrm{s}^{-1}$ through the use of an iodine absorption cell (Valenti et al. 1995; Butler et al. 1996) that provides wavelength calibration from 5000 to $6200 \AA$. These data have been successfully used over many years to detect planetary-mass companions to nearby stars (Butler et al. 2006; Wittenmyer et al. 2017). The AAT dataset for HD 118475 consists of 11 measurements acquired over a time baseline of $\sim 12$ years. These data are shown in Table 1.

To fit the RV data, we used a modified version of the RadVel package developed by Fulton et al. (2018). The
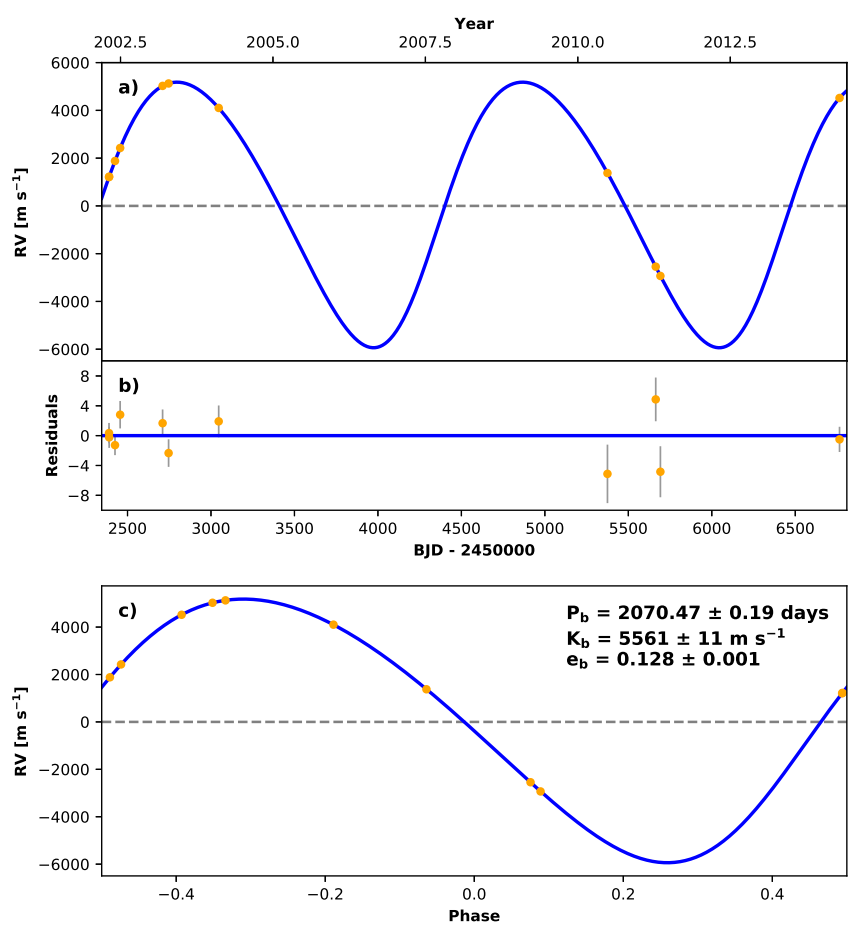

Figure 1. AAT RV data for HD 118475 (shown as yellow points) and the best-fit model (shown as a blue solid curve). The unphased data with residuals are shown in the top panel, and the phased data are shown in the bottom panel.

RadVel code was originally designed for only planetary mass companions because of approximations related to the mass and semi-major axis calculations. Our modification of the RadVel code allows for more massive companions by including the mass of the secondary in the equations that extract the companion minimum mass and semi-major axis from the Keplerian orbital parameters. This modification to RadVel allowed us to calculate the correct mass and orbital semi-major axis of the companion. Shown in Figure 1 are the unphased data with residuals (top panel) and the phased data (bottom panel) along with the best-fit model (solid curve). An offset of $1883.0 \pm 5.6 \mathrm{~m} \mathrm{~s}^{-1}$ was applied to the data during the fit to set the zero-point to the mean value of the model. The extracted companion parameters from the RV fit are shown in Table 2, where $T_{c}$ is the time of inferior conjunction. Also shown in Table 2 are the host star properties from the Spectroscopic Properties of Cool Stars (SPOCS) catalog (Valenti \& Fischer 2005) and the derived companion properties of minimum mass and semi-major axis. Note that the uncertainties on the mass of the secondary are primarily correlated with the mass uncertainties of the host star. 
Table 1. HD 118475 AAT Radial Velocities

\begin{tabular}{ccc}
\hline \hline $\begin{array}{c}\text { Date } \\
(\mathrm{BJD}-2450000)\end{array}$ & $\begin{array}{c}\mathrm{RV} \\
\left(\mathrm{m} \mathrm{s}^{-1}\right)\end{array}$ & $\begin{array}{c}\sigma \\
\left(\mathrm{m} \mathrm{s}^{-1}\right)\end{array}$ \\
\hline 2389.12939 & -675.97 & 1.36 \\
2390.06030 & -658.42 & 1.41 \\
2425.04517 & -1.36 & 1.34 \\
2455.95264 & 542.14 & 1.84 \\
2710.18823 & 3142.47 & 1.84 \\
2746.11157 & 3243.84 & 1.85 \\
3046.20020 & 2219.17 & 2.13 \\
5374.98975 & -506.48 & 3.92 \\
5664.16113 & -4427.03 & 2.93 \\
5692.07959 & -4815.68 & 3.42 \\
6765.16699 & 2636.41 & 1.69 \\
\hline
\end{tabular}

Table 2. HD 118475 System Properties

\begin{tabular}{|c|c|}
\hline Parameter & Value \\
\hline \multicolumn{2}{|l|}{ Host Star } \\
\hline$V$ & 6.97 \\
\hline$d(\mathrm{pcs})$ & 32.9 \\
\hline$M_{\star}\left(M_{\odot}\right)$ & $1.12 \pm 0.11$ \\
\hline$T_{\text {eff }}(\mathrm{K})$ & $5898 \pm 44$ \\
\hline $\log g$ & $4.36 \pm 0.06$ \\
\hline$[\mathrm{Fe} / \mathrm{H}]$ & $0.07 \pm 0.03$ \\
\hline \multicolumn{2}{|c|}{ Companion Measured } \\
\hline$P$ (days) & $2070.47_{-0.2}^{+0.19}$ \\
\hline$T_{c}(\mathrm{BJD})$ & $2455507.9_{-1.1}^{+1.0}$ \\
\hline$e$ & $0.128 \pm 0.001$ \\
\hline$\omega(\operatorname{deg})$ & $237.7 \pm 0.3$ \\
\hline$K\left(\mathrm{~ms}^{-1}\right)$ & $5561 \pm 11$ \\
\hline \multicolumn{2}{|c|}{ Companion Derived } \\
\hline$M_{B} \sin i\left(M_{\odot}\right)$ & $0.445 \pm 0.025$ \\
\hline$a(\mathrm{AU})$ & $3.69 \pm 0.11$ \\
\hline \multicolumn{2}{|c|}{ Measurements and Model } \\
\hline$N_{\text {obs }}$ & 11 \\
\hline $\operatorname{rms}\left(\mathrm{ms}^{-1}\right)$ & 2.93 \\
\hline$\chi_{\text {red }}^{2}$ & 3.27 \\
\hline
\end{tabular}

\section{DIRECT IMAGING OBSERVATIONS}

DSSI is a dual-channel speckle imaging system in which each channel records speckle patterns in narrowband filters with central wavelengths of 692 and $880 \mathrm{~nm}$. The instrument is described in more detail by Horch et al. (2009), including specifics regarding data reduction and performance statistics. HD 118473 was observed using DSSI on Gemini-South during the night of June 7, 2017 (BJD = 2457911). Shown in the left panels of Figure 2 are the DSSI images using the $692 \mathrm{~nm}$ (top) and $880 \mathrm{~nm}$ (bottom) filters, where the contrast of the images have been fixed to maximize the dynamic range of flux due to possible companions. The limiting magnitude curves are shown in the right panels of Figure 2 for the $692 \mathrm{~nm}$ (top) and $880 \mathrm{~nm}$ (bottom) filters. These limiting magnitude figures plot the magnitude difference between local maxima and minima as a function of the separation from the primary star and include a cubic spline interpolation (red solid line) of the $5 \sigma$ detection limit for the full range of angular separations represented.

According to the orbital solution shown in Table 2, the DSSI observation occurred $\sim 333$ days past the passage of inferior conjunction. Using the formalism developed by Kane (2013) and Kane et al. (2018), we calculate the angular separation between the primary star and the companion at the time of DSSI observation to be $\sim 0.09^{\prime \prime}$, compared with the maximum angular separation for the companion of $0.11^{\prime \prime}$. The resulting detection limits of the companion at the time of observation are discussed in detail in the following section.

\section{THE WHITE DWARF HYPOTHESIS}

The imaging observations detailed in Section 3 should be more than capable of resolving a main-sequence companion of the mass required to explain the RV observations described in Section 2. We demonstrate this by modeling the luminosity evolution of the primary and a $0.445 M_{\odot}$ (M dwarf) secondary using the MESA Isochrones and Stellar Tracks (MIST) models (Choi et al. 2016; Dotter 2016). As shown in the left panel of Figure 3, at the current age of the system ( 4.1 Gyr, Valenti \& Fischer 2005), an M dwarf companion would be 71.4 times less luminous than the primary (corresponding to a magnitude difference of 4.63 magnitudes). With this result, we can confidently rule out the hypothesis that the companion is an early $\mathrm{M}$ dwarf to the $2.37 \sigma$ level in the $692 \mathrm{~nm}$ observation, and to the $3.32 \sigma$ level in the $880 \mathrm{~nm}$ observation (see the right panel of Figure 3), for the angular separation at the time of observation (see Section 3).

At first glance, the uncertainty in the mass of the companion might appear to offer a ready explanation for the 

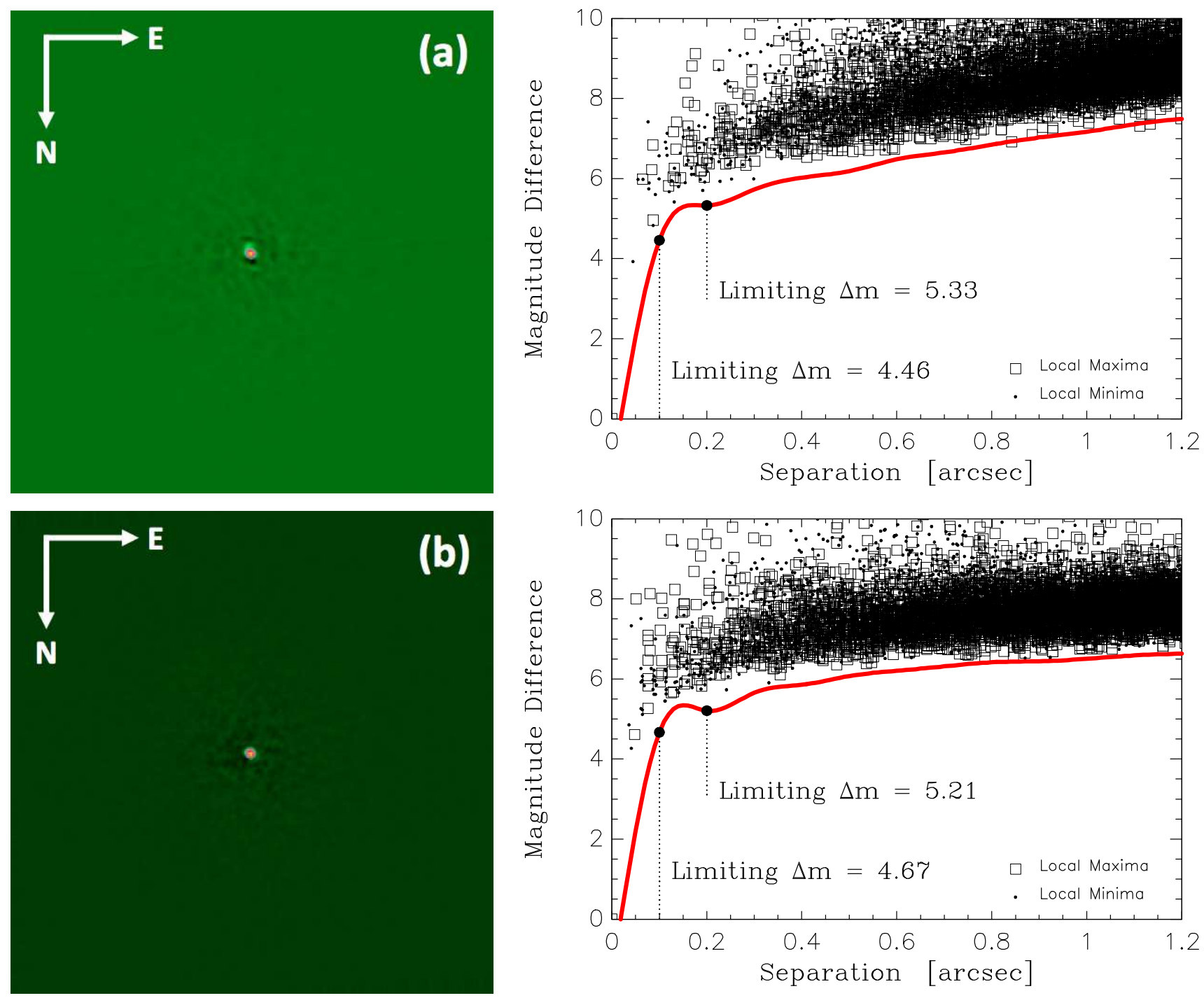

Figure 2. The DSSI images (left) and detection limit plots (right) for the $692 \mathrm{~nm}$ (top) and $880 \mathrm{~nm}$ (bottom) bandpasses respectively. The images on the left are marked as (a) and (b) for $692 \mathrm{nnm}$ and $880 \mathrm{~nm}$ respectively. The field-of-view for the camera is $2.8 \times 2.8^{\prime \prime}$, north is down, and east is to the right. The limiting magnitude data shown in the right panels include cubic spline interpolations (red solid line) of the $5 \sigma$ detection limit.

non-detection. Since the luminosity of a given star is, to first order, proportional to its mass to the fourth power (Smith 1983), one might think that a relatively small reduction in the mass of the secondary could be sufficient to lower its luminosity to a level where it would not be detectable. However, the uncertainty in the mass of the secondary is strongly correlated with the uncertainty in the mass of the primary. In fact, the derived uncertainty in the secondary's mass is smaller, as a fraction of the total mass, than the uncertainty on the primary. Therefore, the only way to reduce the companion's mass would be to reduce the primary's mass. In doing so, the contrast ratio between them would still be such that the companion would be readily detectable in imaging observations at $880 \mathrm{~nm}$.
Interestingly, the fact that the companion must be a compact object in turn means that the system cannot be edge-on to our line of sight. Given the age of the system ( $\sim 4 \mathrm{Gyr})$, we can place limits on the minimum mass that such a compact companion could have, on the basis that such a body must have passed through the entirety of its main-sequence evolution. We modeled the evolution of stars of varying mass, assuming that the initial metallicity of the companion matched that of the primary $([\mathrm{Fe} / \mathrm{H}]=0.07)$, using MIST. As a function of initial progenitor mass, we noted the age at which the post-main sequence mass-loss rate declined to approximately zero. This age was coincident with the beginning of the white dwarf cooling phase. In this manner, we determined that the lowest-mass progenitor for 

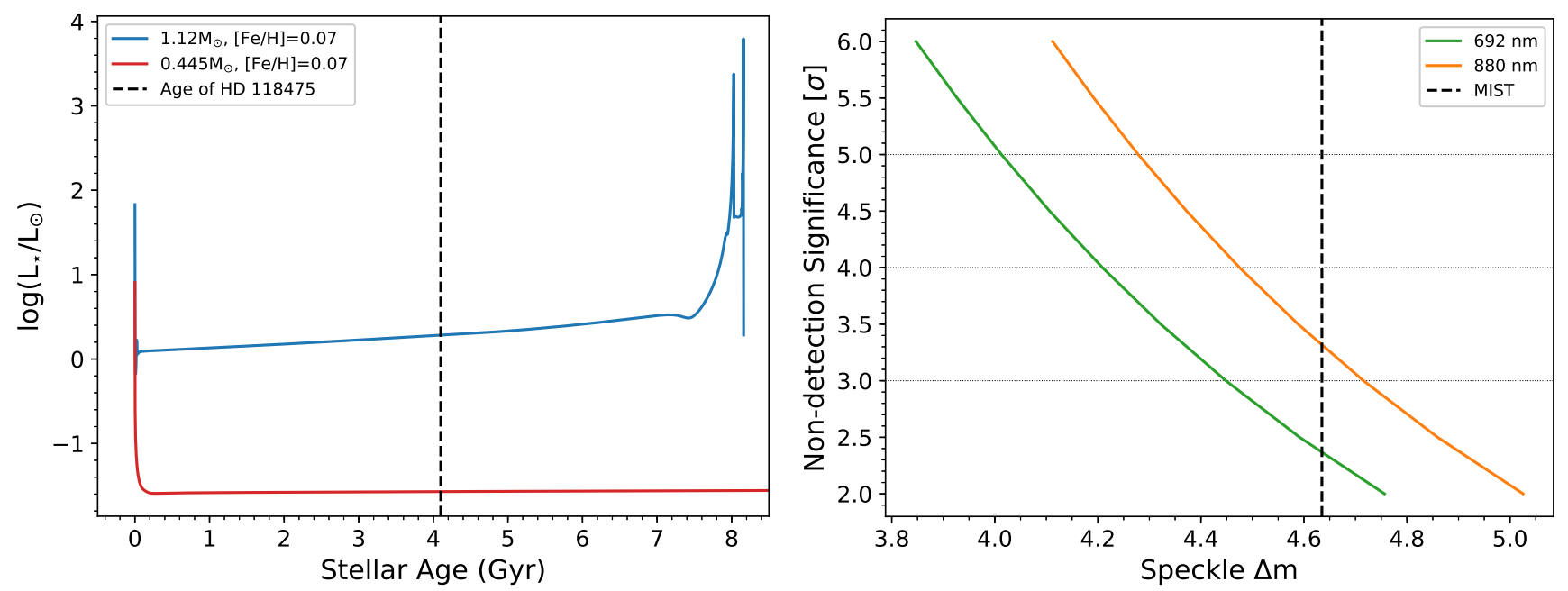

Figure 3. Left: evolutionary tracks for the primary and the secondary-assuming the secondary is an M dwarf-from MIST. The system age ( 4.1 Gyr, Valenti \& Fischer 2005) is indicated by the dashed line. At this age, the M dwarf secondary would be 4.63 magnitudes fainter than the primary. Right: the non-detection significance inferred from the $692 \mathrm{~nm}$ and $880 \mathrm{~nm}$ observations. The dashed line is drawn at 4.63 magnitudes, which is ruled out to a significance of $2.37 \sigma$ and $3.32 \sigma$ for 692 and $880 \mathrm{~nm}$, respectively.

a white dwarf companion that would have completed its evolution within the 4.1-Gyr age of the system would be a $1.38 M_{\odot}$ star. According to the MIST tracks shown in Figure 3, such a star would leave a white dwarf of mass $0.559 M_{\odot}$. Comparing this mass with the $M_{B} \sin i$ of $0.445 M_{\odot}$ allows us to estimate the range of orbital inclinations that are allowed for our solution. We find that orbital inclinations between $90^{\circ}$ (edge-on) and $52.8^{\circ}$ are excluded by the compact nature of the white dwarf. Thus, the system is not old enough to have produced such a low-mass compact companion. There is obviously a chance that the unseen companion could be more massive still. A white dwarf can be no more massive than the Chandrasekhar limit of $1.4 M_{\odot}$. If the companion is truly a white dwarf, this means that the inclination cannot be less than $18.5^{\circ}$, as such an inclination would require the mass to exceed the Chandrasekhar limit.

This opens up the interesting, but unlikely possibility that the unseen companion is either a neutron star or black hole. Our observations do not rule out such an eventuality. We note that the theoretical maximum mass for a neutron star is of order $\sim 3 M_{\odot}$ which corresponds to an orbital inclination for the system of $\sim 8.5^{\circ}$, beyond which the unseen companion must be a black hole.

A fascinating aspect of the system is the significantly non-zero eccentricity of the compact companion. Given the age of the system and the reasonable assumption that the progenitor of the compact object had a higher initial mass, conservation of angular momentum would require that the progenitor had a smaller separation from the current primary than is currently observed. In that case, one may presume that tidal circularization would have produced a circular orbit before the progenitor departed from the main-sequence. To estimate the range of semi-major axes over which we can expect circularization to have occurred, we used the expression for the turbulent dissipation circularization timescale provided in Equation (4.13) by Zahn (1977). For simplicity, we assumed a mass ratio of unity, a primary radius of $1 R_{\odot}$, primary mass of $1.12 M_{\odot}$ (see Table 2 ), primary luminosity of $1 L_{\odot}$, and an apsidal motion constant for the second harmonic of $k_{2}=0.01444$ for a polytropic index of $n=3$ (Brooker \& Olle 1955). Based on these values, we find that the main-sequence progenitor of the compact companion would not have become tidally circularized before leaving the main-sequence unless it was located within $\sim 0.1$ AU of the current primary star, which is unlikely given its present semi-major axis of 3.69 AU. However, departure from the main-sequence into the red giant phase can have a dramatic effect on the tidal circularization timescale for binary systems. Using the methodology of Verbunt \& Phinney (1995) and our estimated minimum mass of the progenitor $\left(1.38 M_{\odot}\right)$, we estimate that tidal circularization of the current primary by the white dwarf progenitor would have occurred out to orbital periods of $\sim 1550$ days, or 3.55 AU. However, outspiralling of the progenitor due to mass loss during the transition from the asymptotic giant branch to a white dwarf means that the stellar components were originally much closer together. Given that the current semi-major axis of the system is $3.69 \mathrm{AU}$ (see Table 2) and the original separation of the system would have been much smaller, tidal circularization should certainly have occurred. Thus, the eccentricity of the companion is unlikely to have been inherited from its orbit whilst 
on the main-sequence. The current orbit may have been perturbed by a close stellar encounter or additional companion in a wide orbit.

A particular issue that is raised by the discovery of this compact companion is the completeness of white dwarf surveys in the solar neighborhood. For example, it was found by Tremblay et al. (2014) that the results of various white dwarf surveys are consistent with each other, but inconsistent with the expected population of white dwarfs based upon the demographics and age of nearby stars. This suggests that there remains a large fraction of stars for which their true binarity remains unresolved. The observational methods used in this work highlight an additional avenue through which the current dearth of known white dwarfs around nearby stars may be mitigated.

In the coming years, data from the Gaia spacecraft (Gaia Collaboration et al. 2016) should provide a measurement of the amplitude of the astrometric wobble imposed upon HD 118475 by its unseen companion. Such data will provide an unequivocal answer to the true mass of the companion. The RV observations made by the AAPS constrain the line-of-sight motion of the star, whilst the astrometric data obtained by Gaia will detail the motion at right angles to our line of sight. By combining the two, the true system inclination will be determined, which in turn will precisely constrain the companion mass.

\section{CONCLUSIONS}

Using RV observations of the star HD 118475 that span a period of 11 years, we find evidence of a massive $\left(M_{B} \sin i=0.445 M_{\odot}\right)$ companion moving on a $\sim 2070$ day orbit, corresponding to an orbital semi-major axis of 3.69 AU. Typically, one would assume that such a companion is most likely to be a previously undetected main sequence star, with the calculated mass suggesting an early $\mathrm{M}$ dwarf. We therefore carried out observations of the system using DSSI on Gemini-South. With those observations, we can rule out a main sequence companion to HD 118475 at the $\sim 3.3 \sigma$ level.

The source of the periodic RV signal observed for HD 118475 must therefore be a compact object, most likely a white dwarf based on the range of possible orbital inclinations. By considering the age of the system ( $\sim 4.1$ Gyr), we determine that the minimum mass that such a compact companion could have is $\sim 0.56 \mathrm{M}_{\odot}$. Compact companions with a lower mass can be excluded on the basis that the progenitor required for such a body would not have had time to evolve beyond its mainsequence lifetime. The fact that the companion must be more massive than $\sim 0.56 M_{\odot}$ means that the system's orbital plane cannot be edge-on to the line of sight. Indeed, orbital inclinations greater than $\sim 53^{\circ}$ can be excluded on the basis of the calculated minimum white dwarf mass.

We note that orbital inclinations between $\sim 8^{\circ}$ and $\sim 18^{\circ}$ would suggest that the unseen companion is actually a neutron star, while inclinations less than $\sim 8^{\circ}$ would suggest a black hole. Such outcomes are unlikely, given the scarcity of such massive compact objects, and so the white dwarf hypothesis seems by far the most likely explanation for the non-detection of a companion through direct imaging. In the future, the release of data obtained by the Gaia mission will allow the orbital inclination of the system to be determined with exquisite precision, which, in combination with the existing RV data, will produce a measurement of the companion's true mass. Furthermore, direct imaging experiments with greater sensitivity capabilities are highly encouraged to attempt to detect the unseen companion, and confirm its white dwarf nature. A DA white dwarf has an absolute magnitude of $M_{V} \sim 12$, which results in a required sensitivity of at least $\Delta m_{V} \sim 7$ for a successful detection. More generally, our results serve as an important reminder of the value of long-term RV exoplanet surveys, and suggest that the data from such surveys should be revisited to examine systems for which long-term, high-amplitude trends led to certain targets being abandoned in favor of those more likely to yield exoplanetary detections.

\section{ACKNOWLEDGEMENTS}

This work is based on observations obtained at the Gemini-South Observatory, which is operated by the Association of Universities for Research in Astronomy, Inc., under a cooperative agreement with the NSF on behalf of the Gemini partnership: the National Science Foundation (United States), National Research Council (Canada), CONICYT (Chile), Ministerio de Ciencia, Tecnología e Innovación Productiva (Argentina), Ministério da Ciência, Tecnologia e Inovação (Brazil), and Korea Astronomy and Space Science Institute (Republic of Korea). We acknowledge the traditional owners of the land on which the AAT stands, the Gamilaraay people, and pay our respects to elders past and present. The results reported herein benefited from collaborations and/or information exchange within NASA's Nexus for Exoplanet System Science (NExSS) research coordination network sponsored by NASA's Science Mission Directorate. 
Barnbaum, C., \& Zuckerman, B. 1992, ApJL, 396, L31

Bonfils, X., Delfosse, X., Udry, S., et al. 2013, A\&A, 549, A109

Borucki, W. J., Koch, D., Basri, G., et al. 2010, Science, 327, 977

Brooker, R. A., \& Olle, T. W. 1955, MNRAS, 115, 101

Butler, R. P., Marcy, G. W., Williams, E., et al. 1996, PASP, 108, 500

Butler, R. P., Wright, J. T., Marcy, G. W., et al. 2006, ApJ, 646, 505

Butler, R. P., Vogt, S. S., Laughlin, G., et al. 2017, AJ, 153, 208

Choi, J., Dotter, A., Conroy, C., et al. 2016, ApJ, 823, 102

Crepp, J. R., Gonzales, E. J., Bechter, E. B., et al. 2016, ApJ, 831, 136

Crepp, J. R., Gonzales, E. J., Bowler, B. P., et al. 2018, ApJ, 864,42

Diego, F., Charalambous, A., Fish, A. C., \& Walker, D. D. 1990, in Proc. SPIE, Vol. 1235, Instrumentation in Astronomy VII, ed. D. L. Crawford, 562-576

Dotter, A. 2016, ApJS, 222, 8

Duquennoy, A., \& Mayor, M. 1991, A\&A, 248, 485

Fulton, B. J., Petigura, E. A., Blunt, S., \& Sinukoff, E. 2018, PASP, 130, 044504

Gaia Collaboration, Prusti, T., de Bruijne, J. H. J., et al. 2016, A\&A, 595, A1

Horch, E. P., Veillette, D. R., Baena Gallé, R., et al. 2009, AJ, 137,5057

Kane, S. R. 2013, ApJ, 766, 10

Kane, S. R., Meshkat, T., \& Turnbull, M. C. 2018, AJ, 156, 267

Kane, S. R., Schneider, D. P., \& Ge, J. 2007, MNRAS, 377, 1610

Kane, S. R., Howell, S. B., Horch, E. P., et al. 2014, ApJ, 785, 93
Kane, S. R., Barclay, T., Hartmann, M., et al. 2015, ApJ, 815, 32

Maxted, P. F. L., Marsh, T. R., \& Moran, C. K. J. 2000, MNRAS, 319, 305

Raghavan, D., McAlister, H. A., Henry, T. J., et al. 2010, ApJS, 190,1

Rebassa-Mansergas, A., Ren, J. J., Irawati, P., et al. 2017, MNRAS, 472, 4193

Ricker, G. R., Winn, J. N., Vanderspek, R., et al. 2015, Journal of Astronomical Telescopes, Instruments, and Systems, 1, 014003

Roberts, Jr., L. C., Tokovinin, A., Mason, B. D., et al. 2015, AJ, 149, 118

Smith, R. C. 1983, The Observatory, 103, 29

Tremblay, P.-E., Kalirai, J. S., Soderblom, D. R., Cignoni, M., \& Cummings, J. 2014, ApJ, 791, 92

Valenti, J. A., Butler, R. P., \& Marcy, G. W. 1995, PASP, 107, 966

Valenti, J. A., \& Fischer, D. A. 2005, ApJS, 159, 141

Verbunt, F., \& Phinney, E. S. 1995, A\&A, 296, 709

Wittenmyer, R. A., Tan, X., Lee, M. H., et al. 2014, ApJ, 780, 140

Wittenmyer, R. A., Butler, R. P., Tinney, C. G., et al. 2016, ApJ, 819, 28

Wittenmyer, R. A., Jones, M. I., Horner, J., et al. 2017, AJ, 154, 274

Wittrock, J. M., Kane, S. R., Horch, E. P., et al. 2016, AJ, 152, 149

—. 2017, AJ, 154, 184

Zahn, J.-P. 1977, A\&A, 57, 383 